\title{
OPEN Artificial intelligence enabled automated diagnosis and grading of ulcerative colitis endoscopy images
}

\author{
Reed T. Sutton ${ }^{1}$, Osmar R. Zäiane ${ }^{2,3}$, Randolph Goebel ${ }^{2,3}$ \& Daniel C. Baumgart ${ }^{1,2 \bowtie}$
}

Endoscopic evaluation to reliably grade disease activity, detect complications including cancer and verification of mucosal healing are paramount in the care of patients with ulcerative colitis (UC); but this evaluation is hampered by substantial intra- and interobserver variability. Recently, artificial intelligence methodologies have been proposed to facilitate more objective, reproducible endoscopic assessment. In a first step, we compared how well several deep learning convolutional neural network architectures (CNNs) applied to a diverse subset of 8000 labeled endoscopic still images derived from HyperKvasir, the largest multi-class image and video dataset from the gastrointestinal tract available today. The HyperKvasir dataset includes 110,079 images and 374 videos and could (1) accurately distinguish UC from non-UC pathologies, and (2) inform the Mayo score of endoscopic disease severity. We grouped 851 UC images labeled with a Mayo score of 0-3, into an inactive/mild (236) and moderate/severe (604) dichotomy. Weights were initialized with ImageNet, and Grid Search was used to identify the best hyperparameters using fivefold cross-validation. The best accuracy (87.50\%) and Area Under the Curve (AUC) (0.90) was achieved using the DenseNet121 architecture, compared to $72.02 \%$ and 0.50 by predicting the majority class ('no skill' model). Finally, we used Gradientweighted Class Activation Maps (Grad-CAM) to improve visual interpretation of the model and take an explainable artificial intelligence approach (XAI).

Ulcerative colitis and Crohn's disease, together referred to as inflammatory bowel disease(s) (IBD), are two chronic systemic inflammatory disorders ${ }^{1,2}$. They result from an inappropriate immune response towards the commensal microbiota in genetically susceptible individuals ${ }^{3}$. Ulcerative colitis cannot be cured, requires lifelong medical therapy ${ }^{4}$, and can progress from repeated flare-ups to complete digestive failure ${ }^{5}$.

Endoscopy is paramount in establishing the initial diagnosis, evaluating disease extent or disease activity, assessing disease complications, providing cancer surveillance and can establish a hard endpoint in clinical trials investigating new treatments ${ }^{6,7}$. Therapeutic strategy has evolved towards seeking combined hard endpoints (such as clinical and endoscopic remission) ${ }^{8,9}$. Mucosal healing has been associated with favorable long-term outcomes $^{10,11}$.

However, endoscopic scoring systems for ulcerative colitis are heterogeneous and subjective, with significant inter- and intra-observer variability; and are still not routinely used in clinical practice ${ }^{12}$. Even in randomized controlled trials, there is great variation in their application and interpretation ${ }^{13}$. Standardization of scoring through unbiased remote central reading is an ideal solution, but not feasible in daily clinical practice ${ }^{14}$.

Machine learning (ML), computer vision (CV) and other algorithmic methodologies commonly referred to as artificial intelligence (AI) techniques have shown promise in mostly classic radiologic diagnostic imaging. The available literature suggests that AI models are capable of being as accurate or superior to human experts at certain medical tasks ${ }^{15-17}$.

But application of $\mathrm{AI}$ in the context of inflammatory bowel diseases is in the very early stages ${ }^{18}$. Preliminary evidence suggests that convolution neural networks (CNN) may be useful to classify severity of ulcerative colitis on endoscopic images ${ }^{19-21}$. However, more data and validation are required to inform analysis approaches and algorithm selection. Here, we investigate the ability of deep learning ${ }^{22}$ algorithms to distinguish ulcerative colitis

${ }^{1}$ Division of Gastroenterology, University of Alberta, 130 University Campus, Edmonton, AB T6G 2X8, Canada. ${ }^{2}$ Department of Computing Science, University of Alberta, Edmonton, $A B$, Canada. ${ }^{3}$ Alberta Machine Intelligence Institute, University of Alberta, Edmonton, AB, Canada. ${ }^{\bowtie}$ email: baumgart@ualberta.ca 


\begin{tabular}{|l|l|l|}
\hline GI tract segment & Pathological finding & \# Images \\
\hline Upper & Barret's (2 classes) & 94 \\
\hline Upper & Esophagitis (2 classes) & 663 \\
\hline Lower & Polyps & 1028 \\
\hline Lower & Ulcerative colitis (6 classes) & 851 \\
\hline Lower & Hemorrhoids & 6 \\
\hline
\end{tabular}

Table 1. Number of labelled images for each pathological finding contained in the HyperKvasir dataset.

\begin{tabular}{|l|l|l|}
\hline Mayo grade & Findings & \# Images \\
\hline Grade 0 & Inactive, mucosa has normal vasculature & 0 \\
\hline Grade $0-1^{*}$ & - & 35 \\
\hline Grade 1 & Mild with erythema, decreased vascular pattern, mild friability & 201 \\
\hline Grade $1-2^{\star}$ & - & 11 \\
\hline Grade 2 & Moderate with erythema, absent vascular pattern, mild friability, erosions & 443 \\
\hline Grade $2-3^{*}$ & - & 28 \\
\hline Grade 3 & Severe with spontaneous bleeding and ulcerations & 133 \\
\hline
\end{tabular}

Table 2. Number of images for each ulcerative colitis Mayo score grade contained in the HyperKvasir dataset. ${ }^{\star}$ Ulcerative colitis images in HyperKvasir were classified with partial grades (not a common clinical practice) as they reported higher inter/intraobserver classification on this dataset.

from other classes of intestinal disorders and grade the endoscopic severity of ulcerative colitis using a weakly supervised approach ${ }^{23}$.

\section{Methods}

Dataset. Kvasir is a multi-class dataset from Bærum Hospital in Vestre Viken Health Trust (Norway), collected from 2010 to $2014^{24}$. Kvasir (v2) contains 8000 endoscopic images labelled with eight distinct classes, with approximately 1000 images per class, including ulcerative colitis. The images are assigned only image-level labels, provided by at least one experienced endoscopist as well as medical trainees (minimum of 3 reviewers per label). The images are independent, with only one image per patient.

Standard endoscopy equipment was used. HyperKvasir is an extension of the Kvasir dataset, collected from the same Bærum Hospital from 2008 to 2016, containing 110,079 images, 10,662 of which are labelled with 23 classes of finding ${ }^{25}$. Pathological findings in particular accounted for 12 of 23 classes, which are aggregated and summarized in Table 1 . They can be broadly grouped into Barret's esophagus and esophagitis in the upper GI tract, and polyps, ulcerative colitis, and hemorrhoids in the lower GI tract.

Importantly, the dataset includes 851 ulcerative colitis images which are labelled and graded using the Mayo endoscopic subscore ${ }^{26,27}$ by a minimum of one board certified gastroenterologist and one or more junior doctors or PhD students (total of 3 reviewers per image). The images are in JPEG format, with varying image resolutions, the most common being $576 \times 768,576 \times 720$, and $1072 \times 1920$. Table 2 shows the number of images available for each Mayo grade.

The HyperKvasir study, including the HyperKvasir dataset available through the Center for Open Science we are using here, was approved by Norwegian Privacy Data Protection Authority, and exempted from patient consent because the data were fully anonymous. All metadata was removed, and all files renamed to randomly generated file names before the internal IT department at Bærum hospital exported the files from a central server. The study was exempted from approval from the Regional Committee for Medical and Health Research Ethics-Southeast Norway since the collection of the data did not interfere with the care given to the patient. Since the data is anonymous, the dataset is publicly shareable and complies with Norwegian and General Data Protection Regulation (GDPR) laws. Apart from this, the data has not been pre-processed or augmented in any way.

Training objectives. Two binary classification tasks were formulated from the dataset:

Diagnosis: All pathological findings of ulcerative colitis were grouped along with all other classes of pathological findings in the dataset (Fig. 1a). The problem was formulated as a binary classification task to distinguish UC from non-UC pathology on endoscopic still images.

Grading: Evaluation of disease severity using endoscopic images of UC pathology. Mayo graded image labels were binned into Grades $0-1$ and 2-3. (Fig. 1b) This grouping has been used in previous machine learning studies and for clinical trial endpoints ${ }^{19}$. Therefore, the task was to distinguish inactive/mild from moderate/severe UC.

Data preprocessing. A filter was designed to remove the green picture-in-picture depicting the endoscope. The filter applied a uniform crop to all images, filling in the missing pixels with 0 values, turning them black. 
a) Training Objective \#1: Classification using 2642 pathological endoscopy images from HyperKvasir dataset.

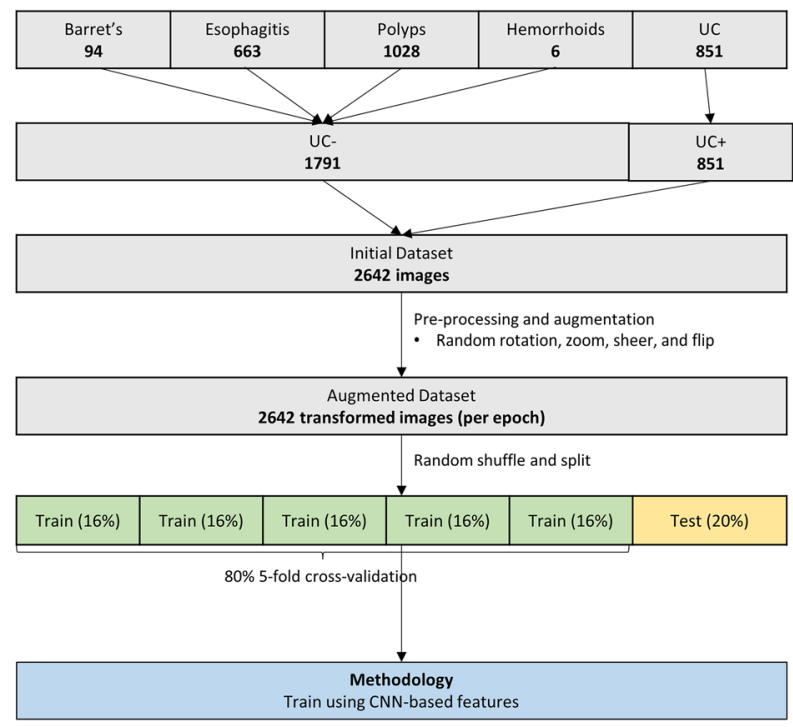

b) Training Objective \#2: Classification using 851 ulcerative colitis images with Mayo grades 0-3 from Hyperkvasir dataset.

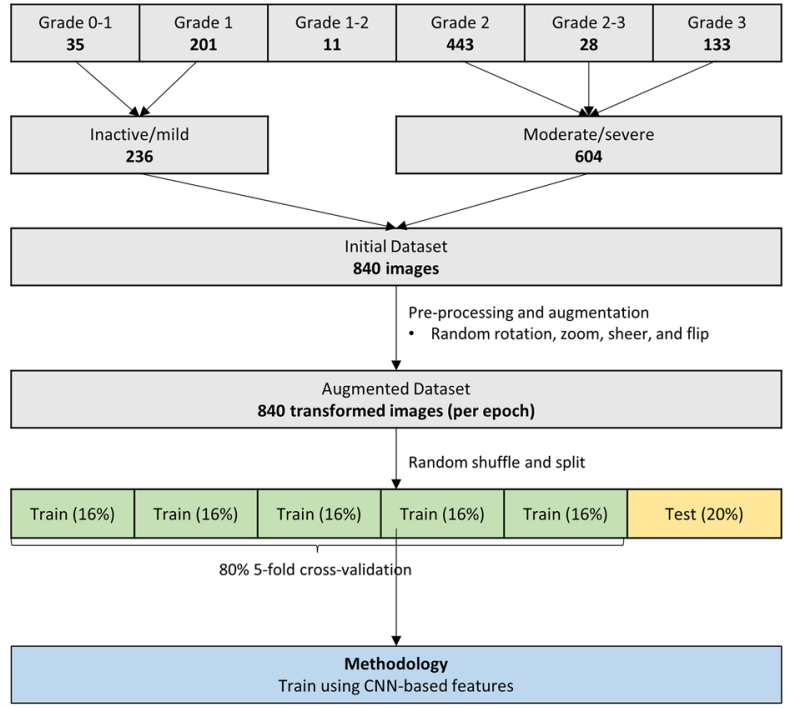

Figure 1. Methods (a) Overview of methods used to train diagnostic classification model of ulcerative colitis from multi-class endoscopic images on Kvasir datasets. (b) Overview of methods used to train diagnostic model for endoscopic grading of ulcerative colitis on HyperKvasir dataset.

Source images were then normalized to $[-1,1]$ and downscaled to $299 \times 299$ resolution using bilinear resampling. Images underwent random transformations of rotation, zoom, sheer, vertical and horizontal flip, using a set seed. Image augmentation was only applied to training set images (not validation or test set), inside each fold of the fivefold cross-validation.

Model generation. There are a growing variety of machine learning frameworks that could provide the foundation for our study. Our choices here acknowledge the current dominance of deep neural network methods, despite the emerging challenges of explainability (explainable artificial intelligence $=\mathrm{XAI}$ ) and trust in practical clinical implementation ${ }^{41}$. Most of our choices use the most popular method for classifying images (convolutional neural networks), whose major differences lie in their depth of layering (50-160) and recorded dimensionality of annotated relationships amongst segments of images (up to 2048).

The following four different $\mathrm{CNN}$ architectures were tested on the Kvasir dataset:

- Pre-trained InceptionV3, a 159-layer CNN. The output of InceptionV3 in this configuration is a 2048-dimensional feature vector ${ }^{28}$

- Pre-trained ResNet50, a Keras implementation of ResNet50, a 50-layer CNN which uses residual functions that reference previous layer inputs ${ }^{29}$.

- Pre-trained VGG19, a Keras implementation of VGG which is a 19 layer CNN developed by Visual Geometry Group $^{30}$.

- Pre-trained DenseNet121, a Keras implementation of DenseNet with 121 layers $^{31}$.

All pre-trained models were TensorFlow implementations initialized using ImageNet weights ${ }^{32}$.Training was performed end-to-end with no freezing of layers. All models performed a final classification step via a dense layer with one node. Sigmoid activation was used at this final dense layer, with binary cross entropy for the model's loss function.

Validation framework. For both classification tasks, the final dataset was randomly shuffled and split into training and validation sets in a 4:1 ratio, where $80 \%$ images were used for fivefold cross-validation and $20 \%$ unseen images were used for evaluating model performance. The best model from each fold were combined and used as the final model for prediction on the test set.

Hyperparameters tuning. Hyperparameters were fine-tuned using Grid Search, where the search space included the following parameters: optimizers: Adam, Stochastic Gradient Descent (SGD), learning rate: 0.01, 0.001, 0.0001; momentum (for SGD): 0, 0.5, 0.9, 0.99. For all models, training phases consisted of 20 epochs with batch size of 32 .

Evaluation metrics. Models were evaluated using accuracy, recall, precision, and F1-scores. As a binary classification problem, confusion matrices and ROC curves were used to visualize model performance. 


\begin{tabular}{|l|l|l|l|l|l|}
\hline \multirow{2}{*}{ Model } & \multicolumn{4}{|l|}{ Metrics } \\
\cline { 2 - 6 } & ACC & SEN & SPEC & F1 & AUC \\
\hline InceptionV3 & 97.92 & 98.61 & 96.47 & 96.76 & 0.9978 \\
\hline ResNet50 & 98.11 & 98.33 & 97.65 & 97.08 & 0.9958 \\
\hline VGG19 & 98.49 & 98.61 & 98.24 & 97.66 & 0.9988 \\
\hline DenseNet121 & 98.30 & 98.89 & 97.06 & 97.35 & 0.9990 \\
\hline Majority class & 67.86 & 100 & 0 & 48.64 & 0.5 \\
\hline
\end{tabular}

Table 3. Evaluation metrics of candidate models for ulcerative colitis diagnosis (binary classification task \#1).

Explainability analysis (XAI). To provide visual explanation of what the models are learning, we chose the Gradient-weighted Class Activation Mapping (Grad-CAM) technique ${ }^{33}$. Grad-CAM produces a heatmap for each model output, showing which part(s) of the image the model is using to make predictions (produces the strongest activation). The heatmap is a course localization map produced by using gradient information flowing into the last convolutional neural network layer, to assign importance values to each neuron.

We also had an experienced gastroenterologist (D.C.B.) annotate and highlight the regions of interest in representative images to provide a comparison with the regions of interest generated by the heatmaps.

Software implementation. Model building was performed and figures created was done using TensorFlow and Keras packages ${ }^{32}$ in Python 3.6.9, run on Google Colab (https://research.google.com/colaboratory/) notebook.

\section{Results}

Model performance. In comparing ulcerative colitis with non-ulcerative colitis endoscopic pathologies, all four of our CNN models achieved very high predictive accuracy in all experiments. Table 3 shows the evaluation metrics performed on the test dataset for each model. The highest AUROC of 0.999 was achieved with DenseNet121, however this did not achieve statistical significance with respect to all other model architectures having extremely high AUROCs (Fig. 2).

In comparing endoscopic remission (Mayo subscore of 0 or 1 ) with moderate to severe active disease ( 2 or 3 ), based on the US FDA definition ${ }^{34}$, all models achieved varying prediction accuracy. Table 4 shows the evaluation metrics performed on the test dataset for each model. The highest AUROC was achieved with DenseNet121, however this did not achieve statistical significance when compared to InceptionV3 results. On the other hand, the shallower CNNs (ResNet50, VGG19), were unable to achieve better accuracy than majority class prediction. AUROC curves are shown in Fig. 3 for the four different CNN architectures.

Explainability analysis (XAI). Gradient-weighted class activation heatmaps for each of the two classification tasks, using DenseNet121 architecture, are shown in Fig. 4 (diagnosis objective) and Fig. 5 (grading objective). The images shown are examples where the model predictions were correct, one for each class (positive or negative). The color scale is from red to orange to blue where red indicates the strongest activation and blue weaker activation.

We note that there can be ambiguity in the heat map indications, compared to the expert analysis. For instance, in Fig. 5B, the heatmaps correctly show the model was activated by fibrin covered ulceration. However, in Fig. $5 \mathrm{~A}$ activation occurs in the most poorly illuminated portion of the image, thus indicating the model is not using the same information that a human would use to make the classification.

\section{Discussion}

We were able to achieve moderate to good performance in mild vs. moderate-to-severe UC on a relatively small public dataset of endoscopy images. This is remarkable given that images having global (image level) labels (Mayo endoscopic subscores), typically require larger datasets to perform well. By approaching the problem as a binary classification problem, the large differences in bowel wall texture seen between inactive/mild and severe cases might have been easier for the model to distinguish.

We were also able to achieve a high accuracy at distinguishing non-ulcerative colitis endoscopic pathologies from ulcerative colitis. However, it should be noted that this problem and the set of endoscopic pathologies modelled do not represent a major clinical challenge. For example, the dataset included Barret's esophagus and esophagitis which are found upon endoscopic examination of the upper gastrointestinal tract (i.e., at an esophagogastroduodenoscopy instead of a colonoscopy-examination of the lower gastrointestinal tract). Therefore, for future studies a more appropriate comparator would be lower gastrointestinal tract pathologies at colonoscopies such as diverticulosis, diverticulitis, microscopic colitis, infectious colitis, or pseudomembranous colitis.

Transfer learning with ImageNet weights is a relatively common approach which has shown success in many medical imaging domains ${ }^{35}$. Particularly for smaller datasets, the pre-learned weights on lower layers augments model training, as they are more general features, while the upper layer weights need to become specific to the training task. In this study, end-to-end training was able to achieve good results, but if not, freezing of the lower layers (known as 'fine-tuning') could be considered. 

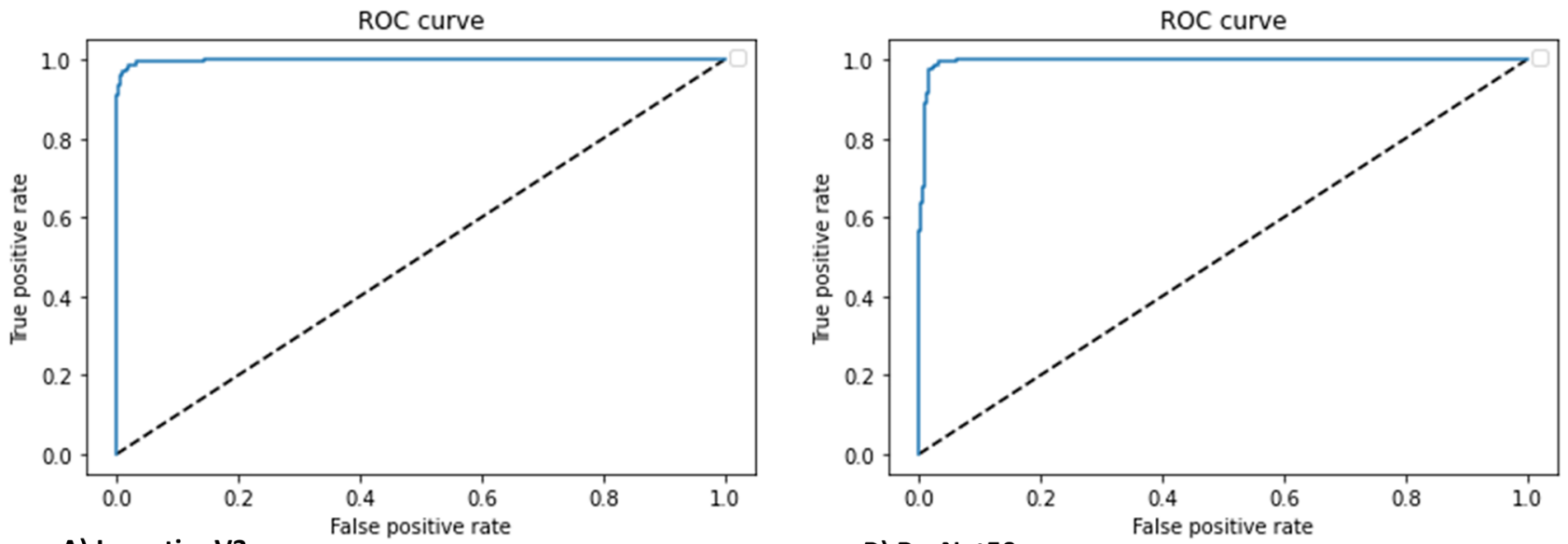

A) InceptionV3

B) ResNet50
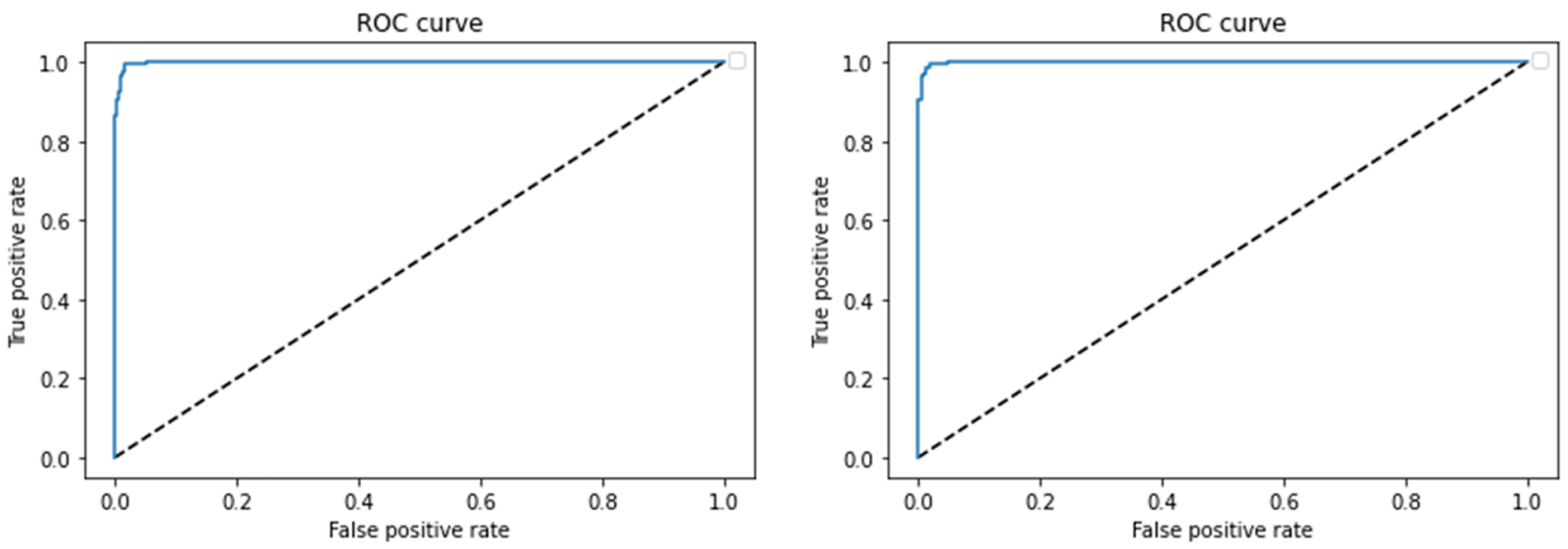

C) VGG19

D) DenseNet121

Figure 2. $\mathrm{CNN}$ discrimination between ulcerative colitis and non-ulcerative colitis pathologies had an area under the receiver operating curve (AUROC) of $>0.99$ with all model architectures (InceptionV3, ResNet50, VGG19, DenseNet121. Dashed lines represent a nondiscriminatory AUROC (0.5).

\begin{tabular}{|l|l|l|l|l|l|}
\hline \multirow{2}{*}{ Model } & \multicolumn{4}{|l}{ Metrics } \\
\cline { 2 - 6 } & ACC & SEN & SPEC & F1 & AUC \\
\hline InceptionV3 & 84.52 & 68.90 & 90.90 & 89.43 & 0.90 \\
\hline ResNet50 & 72.02 & 0 & 100 & 83.74 & 0.66 \\
\hline VGG19 & 73.81 & 6 & 100 & 84.61 & 0.83 \\
\hline DenseNet121 & 87.50 & 79.00 & 91.00 & 91.29 & 0.90 \\
\hline Majority class & 72.02 & 0 & 100 & 83.73 & 0.50 \\
\hline
\end{tabular}

Table 4. Evaluation metrics of candidate models for ulcerative colitis disease activity prediction (binary classification task \#2).

Several other studies have investigated automated UC grading with endoscopic images/videos, and have found similar, promising results. Takenaka et al. (2020) deployed an Inceptionv3 network on 40,000+images from 2012 patients $^{21}$. They achieved 0.945 AUC for predicting endoscopic remission, although they used a total UCEIS score of 0 to indicate remission. Yao et al. (2021) developed an automated video analysis system for grading $\mathrm{UC}^{36}$. Their approach differed in that they predicted global whole-video Mayo subscore based on proportions of individual still images exceeding a given Mayo score, by using a template matching grid search algorithm. In high-resolution videos they were able to achieve a classification accuracy of $78 \%$, and $83.7 \%$ in a lower resolution test set, although agreement between CNN and humans was not high. Stidham et al. (2019) deployed an InceptionV3 model on 16,514 images from 3082 patients, to predict remission (Mayo 0/1 vs. May 2/3), achieving 


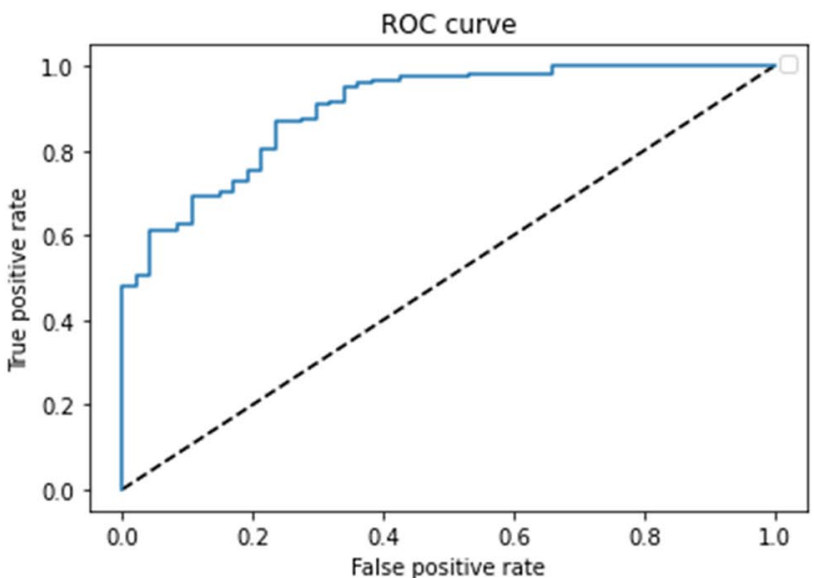

A) InceptionV3

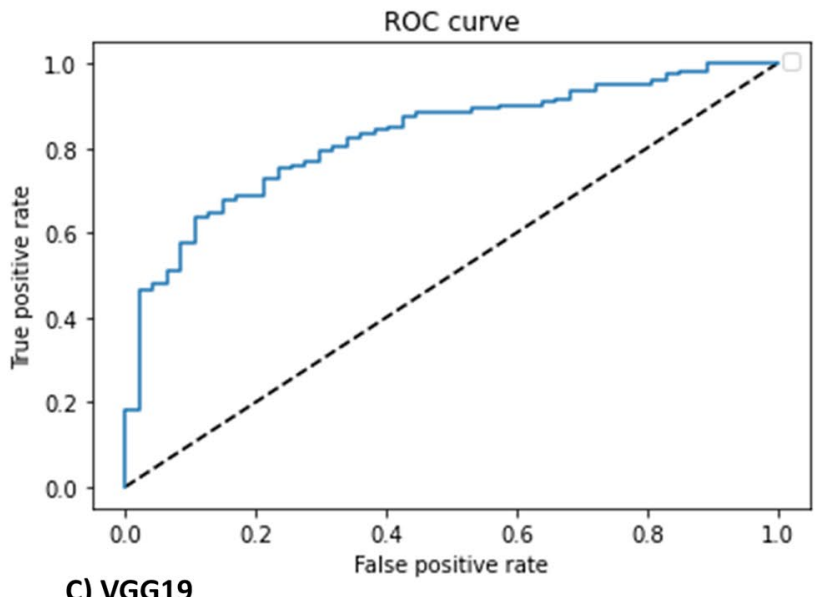

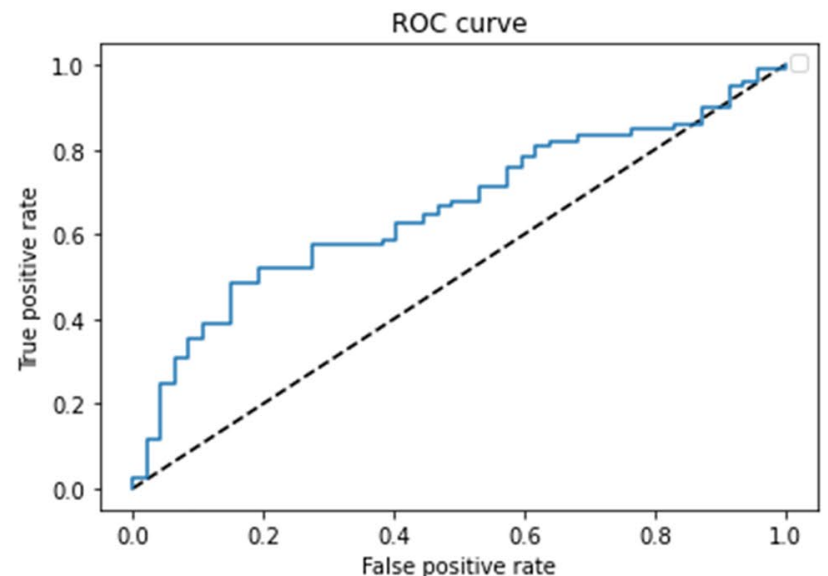

B) ResNet50

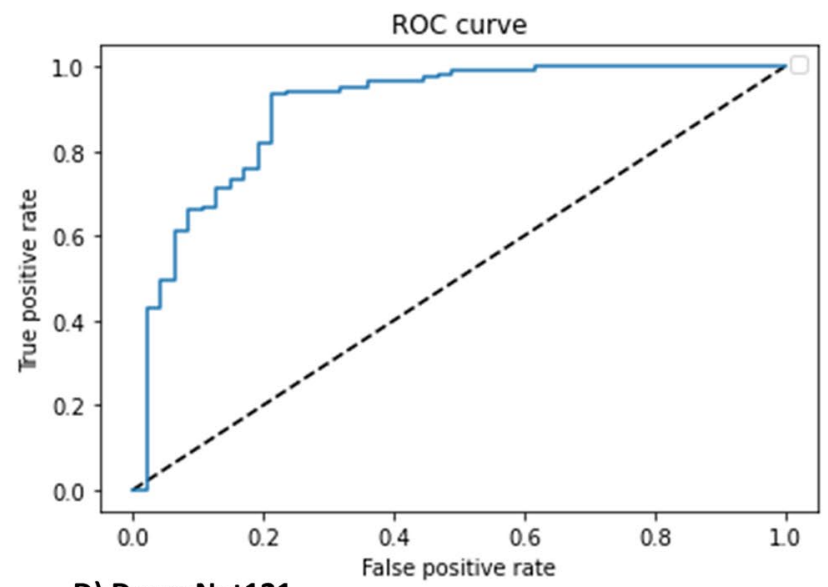

D) DenseNet121

Figure 3. CNN discrimination between endoscopic remission (Mayo 0 or 1) from moderate to severe activity (Mayo 2 or 3) had an area under the receiver operating curve (AUROC) of 0.90 with InceptionV3 (A), 0.66 with ResNet50 (B), 0.83 with VGG19 (C), and 0.90 with DenseNet121 (D). Dashed lines represent a nondiscriminatory AUROC (0.5).

an impressive AUROC of $0.966^{19}$. However few studies have investigated or been successful as multi-class classification using each individual Mayo score as a class. Given a large enough dataset, this should be explored.

Additionally, few prior approaches to automated UC grading have addressed explainability (explainable artificial intelligence $=\mathrm{XAI}$ ), which will be problematic when it comes time to deploy AI models in clinical systems in order to garner physician trust. We attempted to improve explainability of our model by showing representative images of the two classes (positive and negative) along with class activation heatmaps. These heatmaps allow for some speculation as to what patterns the model is identifying to make its prediction. In comparison with the gastroenterologist annotations, we can see that sometimes the heatmaps are identifying clinically representative information, and sometimes they are not. Particularly in Fig. 5B, the heatmaps do suggest the model has learned to recognize fibrin, which is consistent with ulcerative colitis pathology.

As a consequence, we conclude that heatmaps are a good start but remain one of the weaker XAI methods, as they are not semantically driven and can only provide low-level, post-hoc explanations ${ }^{37}$. Other methods such as gradient-based saliency maps, Class Activation Mapping, and Excitation Backpropagation can all be considered in future work ${ }^{38,39}$. 

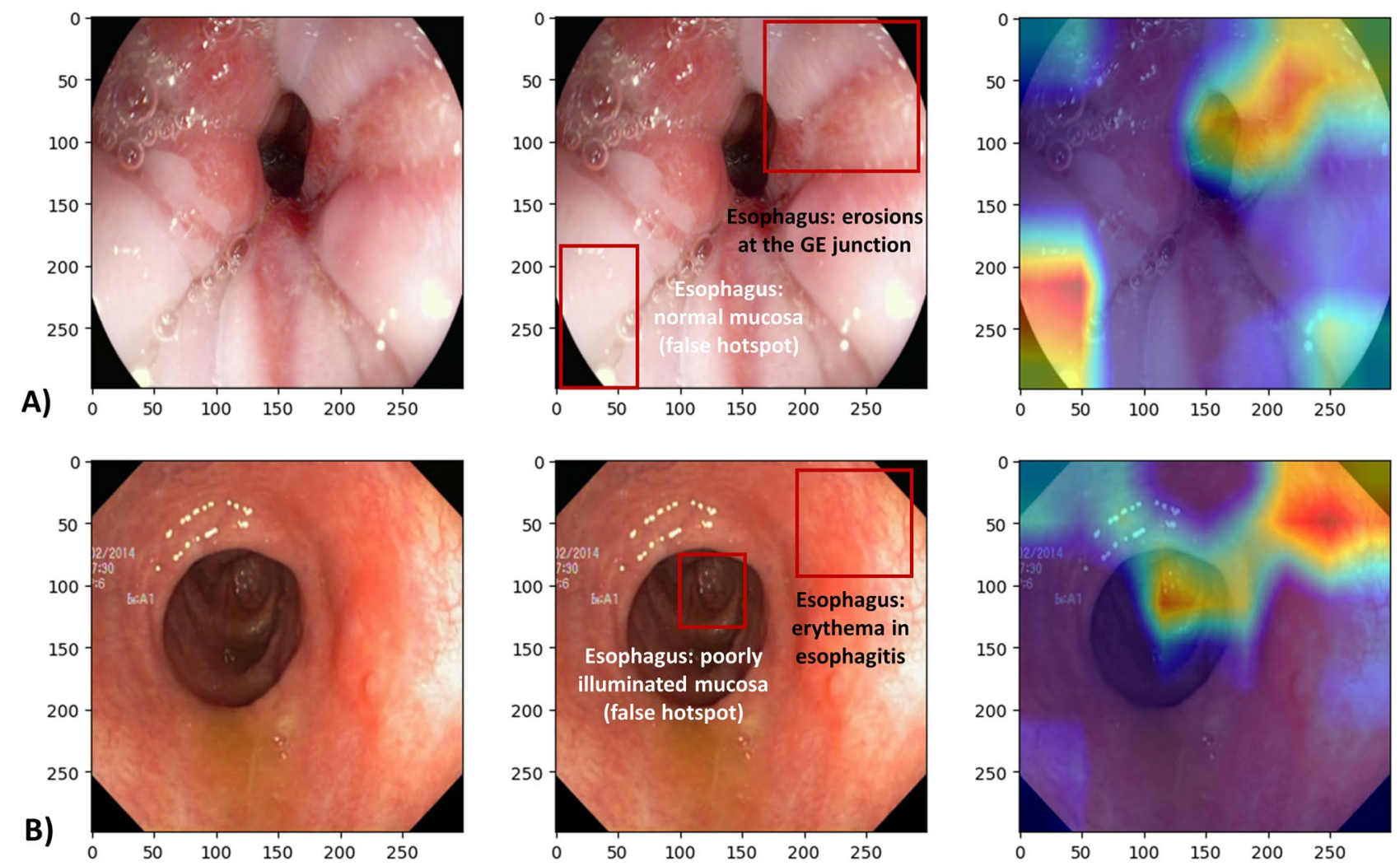

Figure 4. Class activation heatmaps alongside the original endoscopic images. (A) Test image (esophagitis) negative for ulcerative colitis, and (B) Test image positive for ulcerative colitis. Red color indicates the greatest level of activation. Images included are courtesy of the Center for Open Science and carry a CC-By Attribution 4.0 International license: https://osf.io/mh9sj/.

A limitation of this dataset was class imbalance amongst more moderate/severe cases. While we performed stratified splitting, further measures could be taken such as class weighting methods. Ultimately the best solution would be to enrich the dataset. Furthermore, still images present a challenge for Mayo subscore classification, as friability and bleeding may be more difficult to identify in still images than in video. This may have further reduced the true accuracy of the labels provided in the dataset.

Also, two different endoscopes were used, a Pentax (Pentax, Tokyo, Japan) gastroscope for upper endoscopic exams, and an Olympus (Olympus, Tokyo, Japan) colonoscope for the lower, presenting a systematic bias (only for training objective 1 which included both upper and lower pathologies). We consider it unlikely that the model learned to distinguish image features of the scopes themselves based on visual inspection of heatmaps, and equal performance of the model to distinguish ulcerative colitis from upper pathologies, and from polyps, the primary lower pathology. Nonetheless, future work could consider addressing such a bias by performing uniform cropping to remove image outlines, one obvious image feature that could be specific to the endoscope.

In future research we will work with larger and more clinically diverse datasets, and also supplement the feature set with hand-crafted and texture descriptors, for example Color and Edge Directivity Descriptors (CEDD), GLCM, Tamura, or ColorLayout. Such features could be combined with the CNN features by feature fusion at the dense layer (in 'in vivo' models real clinical non-image data could also be added in this step). One such approach found good results by using a red density algorithm (red channel) to correlate with endoscopic and histologic disease ${ }^{40}$. 

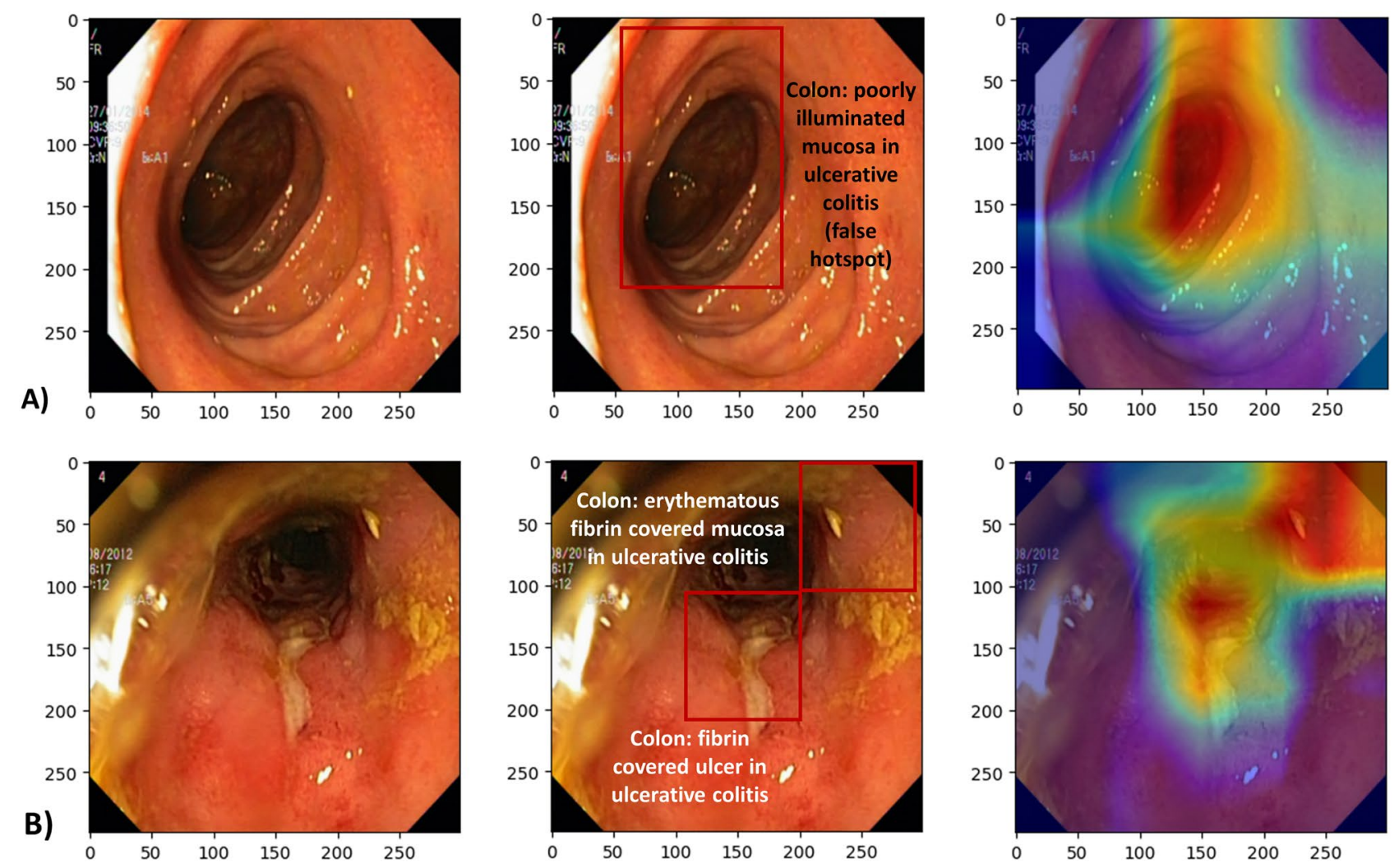

Figure 5. Class activation heatmaps alongside the original endoscopic image for (A) Test image with inactive to mild ulcerative colitis (Mayo 0-1), and (B) Test image positive for moderate to severe ulcerative colitis (Mayo $2-3)$. Red color indicates the greatest level of activation. Images included are courtesy of the Center for Open Science and carry a CC-By Attribution 4.0 International license: https://osf.io/mh9sj/.

\section{Data availability}

The data that support the findings of this study are available from the OSF repository, originally published by Borgli et al. ${ }^{25}$.

\section{Code availability}

The Python and MATLAB source code for this project is available upon reasonable request. Python code was compiled in Google Colab. The images were contained in a zip file in the same Google Drive directory as the hosted code.

Received: 24 September 2021; Accepted: 4 February 2022

Published online: 17 February 2022

\section{References}

1. Baumgart, D. C. et al. Crohn's disease. Lancet 380, 1590-1605. https://doi.org/10.1016/S0140-6736(12)60026-9 (2012).

2. Danese, S. et al. Ulcerative colitis. N. Engl. J. Med. 365, 1713-1725. https://doi.org/10.1056/NEJMra1102942 (2011).

3. Graham, D. B. et al. Pathway paradigms revealed from the genetics of inflammatory bowel disease. Nature 578, 527-539. https:// doi.org/10.1038/s41586-020-2025-2 (2020).

4. Baumgart, D. C. et al. Newer Biologic and Small-Molecule Therapies for Inflammatory Bowel Disease. N Engl J Med 385, $1302-1315$. https://doi.org/10.1056/NEJMra1907607 (2021).

5. Pariente, B. et al. Development of the Crohn's disease digestive damage score, the Lemann score. Inflamm. Bowel Dis. 17, $1415-1422$. https://doi.org/10.1002/ibd.21506 (2011).

6. Sturm, A. et al. ECCO-ESGAR Guideline for Diagnostic Assessment in IBD Part 2: IBD scores and general principles and technical aspects. J. Crohns Colitis 13, 273-284. https://doi.org/10.1093/ecco-jcc/jjy114 (2019).

7. Maaser, C. et al. ECCO-ESGAR Guideline for Diagnostic Assessment in IBD Part 1: initial diagnosis, monitoring of known IBD, detection of complications. J. Crohns Colitis 13, 144-164. https://doi.org/10.1093/ecco-jcc/jjy113 (2019).

8. Peyrin-Biroulet, L. et al. Selecting therapeutic targets in inflammatory bowel disease (STRIDE): determining therapeutic goals for treat-to-target. Am. J. Gastroenterol. 110, 1324-1338. https://doi.org/10.1038/ajg.2015.233 (2015).

9. Turner, D. et al. STRIDE-II: an update on the selecting therapeutic targets in inflammatory bowel disease (STRIDE) initiative of the international organization for the study of IBD (IOIBD): determining therapeutic goals for treat-to-target strategies in IBD. Gastroenterology https://doi.org/10.1053/j.gastro.2020.12.031 (2021).

10. Reinink, A. R. et al. Endoscopic mucosal healing predicts favorable clinical outcomes in inflammatory bowel disease: a metaanalysis. Inflamm. Bowel. Dis. 22, 1859-1869. https://doi.org/10.1097/mib.0000000000000816 (2016). 
11. Froslie, K. F. et al. Mucosal healing in inflammatory bowel disease: results from a Norwegian population-based cohort. Gastroenterology 133, 412-422. https://doi.org/10.1053/j.gastro.2007.05.051 (2007).

12. Vashist, N. M. et al. Endoscopic scoring indices for evaluation of disease activity in ulcerative colitis. Cochrane Datab. Syst. Rev. 1, 011450. https://doi.org/10.1002/14651858.CD011450.pub2 (2018).

13. Yoon, H. et al. Incremental benefit of achieving endoscopic and histologic remission in patients with ulcerative colitis: a systematic review and meta-analysis. Gastroenterology 159, 1262-1275. https://doi.org/10.1053/j.gastro.2020.06.043 (2020).

14. Feagan, B. G. et al. The role of centralized reading of endoscopy in a randomized controlled trial of mesalamine for ulcerative colitis. Gastroenterology 145, 149-157.e142. https://doi.org/10.1053/j.gastro.2013.03.025 (2013).

15. Gulshan, V. et al. Development and validation of a deep learning algorithm for detection of diabetic retinopathy in retinal fundus photographs. JAMA 316, 2402-2410. https://doi.org/10.1001/jama.2016.17216 (2016).

16. Esteva, A. et al. Dermatologist-level classification of skin cancer with deep neural networks. Nature 542, 115-115. https://doi.org/ 10.1038/nature21056 (2017).

17. Hannun, A. Y. et al. Cardiologist-level arrhythmia detection and classification in ambulatory electrocardiograms using a deep neural network. Nat. Med. https://doi.org/10.1038/s41591-018-0268-3 (2019).

18. Bossuyt, P. et al. Scoring endoscopic disease activity in IBD: artificial intelligence sees more and better than we do. Gut 69, 788-789. https://doi.org/10.1136/gutjnl-2019-318235 (2020).

19. Stidham, R. W. et al. Performance of a deep learning model vs human reviewers in grading endoscopic disease severity of patients with ulcerative colitis. JAMA Netw Open 2, e193963-e193963. https://doi.org/10.1001/jamanetworkopen.2019.3963 (2019).

20. Ozawa, T. et al. Novel computer-assisted diagnosis system for endoscopic disease activity in patients with ulcerative colitis. Gastrointest Endosc. 89, 416. https://doi.org/10.1016/j.gie.2018.10.020 (2019).

21. Takenaka, K. et al. Development and validation of a deep neural network for accurate evaluation of endoscopic images from patients with ulcerative colitis. Gastroenterology 158, 2150-2157. https://doi.org/10.1053/j.gastro.2020.02.012 (2020).

22. LeCun, Y. et al. Deep learning. Nature 521, 436-444. https://doi.org/10.1038/nature14539 (2015).

23. Ratner, A. et al. in Weak Supervision: A New Programming Paradigm for Machine Learning (The Stanford AI Lab Blog, 2019). http:// ai.stanford.edu/blog/weak-supervision/

24. Pogorelov, K. et al. KVASIR: A Multi-Class Image Dataset for Computer Aided Gastrointestinal Disease Detection. Proc 8th ACM Multimed Syst Conf, 164-169, doi:https://doi.org/10.1145/3083187.3083212 (2017).

25. Borgli, H. et al. HyperKvasir, a comprehensive multi-class image and video dataset for gastrointestinal endoscopy. Sci. Data 7, 283. https://doi.org/10.1038/s41597-020-00622-y (2020).

26. Schroeder, K. W. et al. Coated oral 5-aminosalicylic acid therapy for mildly to moderately active ulcerative colitis. A randomized study. N. Engl. J. Med. 317, 1625-1629. https://doi.org/10.1056/nejm198712243172603 (1987).

27. Travis, S. P. et al. Reliability and initial validation of the ulcerative colitis endoscopic index of severity. Gastroenterology 145, 987-995. https://doi.org/10.1053/j.gastro.2013.07.024 (2013).

28. Szegedy, C. et al. Rethinking the inception architecture for computer vision. Proc. IEEE Comput. Soc. Conf. Comput. Vis. Pattern Recognit. https://doi.org/10.1109/CVPR.2016.308 (2016).

29. He, K. et al. Deep residual learning for image recognition. Proc. IEEE Comput. Soc. Conf. Comput. Vis. Pattern Recognit. https:// doi.org/10.1109/CVPR.2016.90 (2016).

30. Simonyan, K. et al. Very deep convolutional networks for large-scale image recognition. Int. Conf. Learn Represent (2015). arxiv: 1409.1556v6.

31. Huang, G. et al. Densely connected convolutional networks. Proc. IEEE Comput. Soc. Conf. Comput. Vis. Pattern Recognit. https:// doi.org/10.1109/CVPR.2017.243 (2017).

32. Abadi, M. et al. TensorFlow: a system for large-scale machine learning. Proc 12th USENIX Symp Oper Syst Des Implement, 265-283 (2016). https://www.usenix.org/conference/osdi16/technical-sessions/presentation/abadi.

33. Selvaraju, R. R. et al. Grad-CAM: visual explanations from deep networks via gradient-based localization. Int J Comput Vis 128, 336-359. https://doi.org/10.1109/ICCV.2017.74 (2019).

34. Reinisch, W. et al. Comparison of the EMA and FDA guidelines on ulcerative colitis drug development. Clin. Gastroenterol. Hepatol. https://doi.org/10.1016/j.cgh.2018.10.032 (2018).

35. Shin, H. C. et al. Deep convolutional neural networks for computer-aided detection: CNN architectures, dataset characteristics and transfer learning. IEEE Trans. Med. Imag. 35, 1285-1298. https://doi.org/10.1109/TMI.2016.2528162 (2016).

36. Yao, H. et al. Fully automated endoscopic disease activity assessment in ulcerative colitis. Gastrointest Endosc. 93, 728-736.e721. https://doi.org/10.1016/j.gie.2020.08.011 (2021).

37. Kim, M.-Y. et al. A multi-component framework for the analysis and design of explainable artificial intelligence. Mach. Learn Knowl. Extr. https://doi.org/10.3390/make3040045 (2021).

38. Zhang, J. et al. Top-Down neural attention by excitation backprop. Int J Comput Vis 126, 1084-1102. https://doi.org/10.1007/ s11263-017-1059-x (2018).

39. Simonyan, K. et al. Deep inside convolutional networks: visualising image classification models and saliency maps. Int. Conf. Learn. Represent. arxiv:1312.6034v2 (2014).

40. Bossuyt, P. et al. Automatic, computer-aided determination of endoscopic and histological inflammation in patients with mild to moderate ulcerative colitis based on red density. Gut 69, 1778-1786. https://doi.org/10.1136/gutjnl-2019-320056 (2020).

\section{Acknowledgements}

This work was supported by Canadian Institutes of Health Research (CIHR) Vanier Canada Program, the Canadian Institute for Advanced Research (CIFAR), the Natural Sciences and Engineering Research Council of Canada (NSERC), Alberta Innovates, the University of Alberta and by the Alberta Machine Intelligence Institute (Amii). The opinions expressed in this manuscript are the author's own and do not necessarily reflect those of Alberta Health Services, the University of Alberta or the Government of Alberta.

\section{Author contributions}

R.T.S. and D.C.B. designed the study. R.T.S. analyzed and interpreted the data, coded the models, and wrote the first manuscript draft. O.R.Z, R.G. and D.C.B. interpreted the data and provided critical manuscript revisions. All authors approved the final version for submission and vouch for the accuracy and completeness of the analysis reported.

\section{Competing interests}

The authors declare no competing interests. 


\section{Additional information}

Correspondence and requests for materials should be addressed to D.C.B.

Reprints and permissions information is available at www.nature.com/reprints.

Publisher's note Springer Nature remains neutral with regard to jurisdictional claims in published maps and institutional affiliations.

(c) (1) Open Access This article is licensed under a Creative Commons Attribution 4.0 International License, which permits use, sharing, adaptation, distribution and reproduction in any medium or format, as long as you give appropriate credit to the original author(s) and the source, provide a link to the Creative Commons licence, and indicate if changes were made. The images or other third party material in this article are included in the article's Creative Commons licence, unless indicated otherwise in a credit line to the material. If material is not included in the article's Creative Commons licence and your intended use is not permitted by statutory regulation or exceeds the permitted use, you will need to obtain permission directly from the copyright holder. To view a copy of this licence, visit http://creativecommons.org/licenses/by/4.0/.

(C) The Author(s) 2022 